\title{
Recent Development of Advanced Neutron Detection Technology
}

\author{
Takashi Nakamura* \\ Cyclotron and Radioisotope Center, Tohoku University \\ Aoba, Aramaki, Aoba-ku, Sendai, Japan 980-8578
}

Received: August, 19, 2003

\begin{abstract}
This review paper gives the description on the recent development of various new sophisticated neutron spectrometers. For neutrons of energies beyond $100 \mathrm{MeV}$, the self-TOF detector using the NE102A plastic scintillators, large-scale NE213 organic liquid scintillator, and spallation detectors of $\mathrm{C}$ and $\mathrm{Bi}$ have recently been developed by our group, especially for use in neutron target and shielding experiments. Various neutron spectrometers have also been developed for use in the charged particle and neutron mixed field which is realized in space environment. The following spectrometers are briefly described: 1) Phoswich detector which combines the NE115 plastic scintillator and the NE213 scintillator, 2) Anti-coincidence detector system using $\Delta E$ and $E$ counters, and 3) Bonner sphere (multi-moderator detector) mounted a ${ }^{3} \mathrm{He}$ counter or a pair of ${ }^{6} \mathrm{Li}$ and ${ }^{7} \mathrm{Li}$ glass scintillators.
\end{abstract}

\section{Introduction}

Neutron measurement is in general more difficult than photon and charged-particle measurements for the following reasons:

1) Neutrons interact with only nucleus and the reaction cross sections vary largely with each nucleus and with incident neutron energy, which indicates that the detector response to neutrons will be very complicated.

2) Neutron energies existing in various radiation environments extend from thermal energy to $\mathrm{GeV}$ energy, which requires that the neutron detection must be done over 10 decades of energy region.

3) Neutrons are always accompanied by photons and in space environment by charged particles, which means that the neutron detection necessitates the event discrimination technique between neutron events and other events.

For neutron measurements in nuclear facilities, the multimoderator spectrometer, so-called Bonner Ball, the organic liquid scintillator, and the activation detector have been widely used as spectrometers. But for neutrons of energy above 100 $\mathrm{MeV}$, there have been no suitable spectrometers with reasonable resolutions. Our group recently developed the self-TOF detector using the NE102A plastic scintillators, large-scale NE213 organic liquid scintillator, and spallation detectors of C and $\mathrm{Bi}$ for measuring these high-energy neutrons, which become very important in the radiation environment surrounding the high-energy and high-intensity accelerators because of their strong penetrability.

Inside a spacecraft, there are high-energy protons and heavy ions, and the secondary neutrons are generated by the nuclear reactions between these particles and the material of the spacecraft. Although these secondary neutrons are estimated to contribute to 5 to $30 \%$ of the dose equivalent of intravehicular crews, ${ }^{1,2}$ the information on these neutrons is still very poor, because it is very difficult to discriminate neutron events from dominant charged particle events in the mixed field of neutrons and charged particles in space environment. In the past only two neutron measurements have been done in spacecraft by Russia and USA. ${ }^{1,3}$ Keith et al. ${ }^{1}$ measured a neutron spectrum using a passive Bonner Ball detector (Au Foil). Labakov et

*Corresponding author. E-mail: nakamura@cyric.tohoku.ac.jp. FAX: +81-22-217-7809 al. ${ }^{3}$ measured neutron flux using a Phoswitch-type neutron detector to eliminate charged particles.

Recently, various neutron spectrometers to be used in space environment have been developed and are now being developed with the discrimination technique between charged particle and neutron. Here the outlines of these newly developed neutron spectrometers are described: 1) Phoswich detector which combines the NE115 plastic scintillator and the NE213 scintillator, 2) Anti-coincidence detector system using $\Delta E$ and $E$ counters, and 3) Bonner sphere (multi-moderator detector) mounted a ${ }^{3} \mathrm{He}$ counter or a pair of ${ }^{6} \mathrm{Li}$ and ${ }^{7} \mathrm{Li}$ glass scintillators.

\section{High-Energy Neutron Detectors}

II-1. Large NE213 Organic Liquid Scintillator. An NE213 (or BC501A) organic liquid scintillator coupled with a photo-multiplier is widely used for high energy neutron spectrometry with the unfolding method in target and shielding experiments because of good quality of neutron and photon discrimination, and energy resolution.

The neutron and photon events are usually separated by using two-dimensional graphical plots of slow and total component pulse-heights (Figure 1). When charged particles produced by neutron reactions (mainly recoil protons by the $H(n, n) p$ elastic collision) escape from the NE213 scintillator without complete energy loss, the pulse shapes from high energy neutron events are close to those from gamma-ray events, that is called as a wall effect, and it is impossible to discriminate only the photon events as shown in Figure 1. Both events caused by photons and by escaping charged particles are removed. Therefore, the response functions mentioned below exclude the light outputs caused by the escaping charged particles.

The physical characteristics of the NE213 scintillator, such as response function, detection efficiency and light output yield, are well known in detail for neutrons below $20 \mathrm{MeV}$ through many studies. For higher energy neutron measurement, a large-size NE213 detector, $12.7 \mathrm{~cm}$ ( 5 inch) diam by $12.7 \mathrm{~cm}$ (5 inch) long, coupled with a photomultiplier (Hamamatsu R4144) has been used in our group and a larger $25.4 \mathrm{~cm}$ (10 inch) diam by $25.4 \mathrm{~cm}$ (10 inch) long NE213 is now in preliminary use. With regard to the response functions of the $12.7 \mathrm{~cm}$ diam by $12.7 \mathrm{~cm}$ long NE213 detector for 


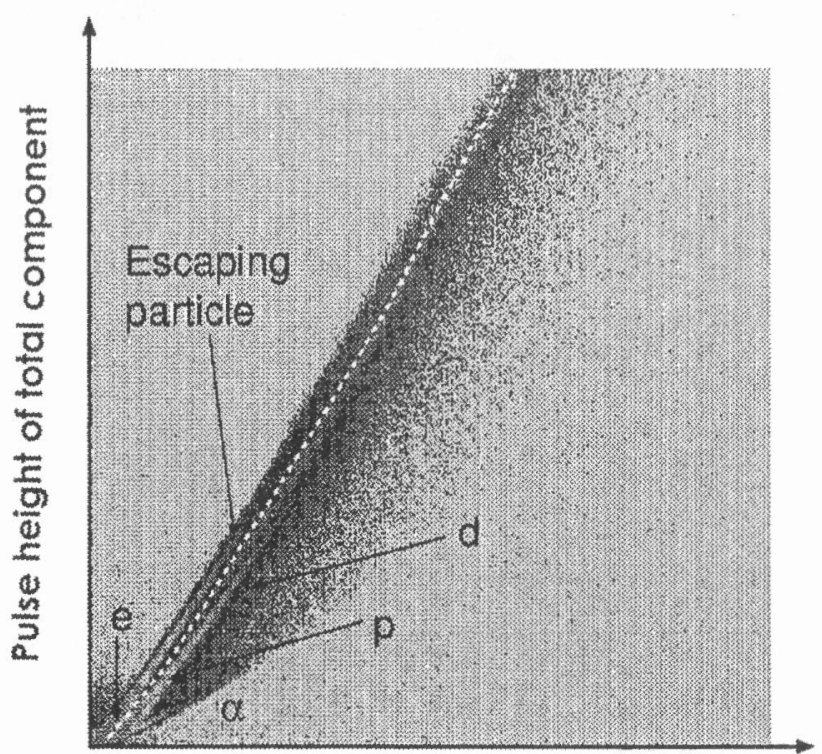

Pulse height of slow component

Figure 1. Two dimensional pulse height scatter plots of the slow component versus total component to identify the particles produced in the NE213 scintillator. The discrimination boundary is indicated by the broken line. The symbol $e$ is the electron produced by photon and $\mathrm{p}, \mathrm{d}$, and $\alpha$, are the proton, deuteron and alpha particle produced by neutrons, respectively.

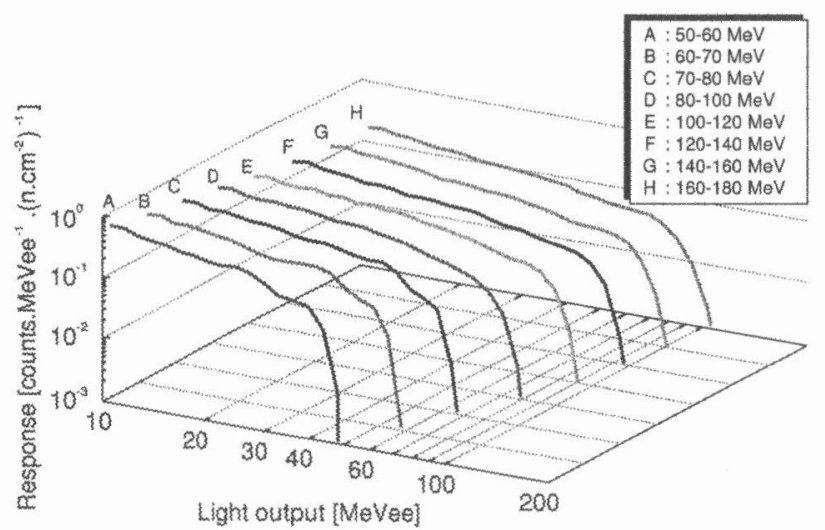

Figure 2. Measured response functions of the NE213 scintillator for $50-60,60-70,70 m 80,80 m 100,100-120,120-140,140-160$, and $160-180 \mathrm{MeV}$ neutrons.

neutrons above $20 \mathrm{MeV}$, Nakao et al. ${ }^{4,5}$ measured them in the energy range up to $206 \mathrm{MeV}$ using the $\mathrm{Li}(\mathrm{p}, \mathrm{n})$ quasi-monoenergetic and white neutron sources at the ring cyclotron facility (RRC) of Institute of Physical and Chemical Research (RIKEN), Japan and Sasaki et al. ${ }^{6}$ extended them up to 800 $\mathrm{MeV}$ using the white neutron source from thick carbon targe bombarded by $400 \mathrm{MeV} /$ nucleon carbon and $800 \mathrm{MeV} /$ nucleon silicon ions at the heavy ion medical accelerator facility (HIMAC) of National Institute of Radiological Science (NIRS), Japan.

The response functions to neutrons with energies between 50 and $800 \mathrm{MeV}$ measured by Sasaki et al. are illustrated in Figures 2 and 3 . The alphabets which appear at the left shoul ders of each spectrum from "A" to "O" correspond to the neutron energy boundaries listed in the figures. It has been hitherto said that the response function does not change above a few hundreds MeV region where the charged particle produced by neutron reactions escape from the scintillator, and indeed, the whole shape and the upper edge of the response functions are very similar to each other beyond $200 \mathrm{MeV}$ neutrons. But as shown in these graphs, a small change of the response function and a further expansion of the upper edge to higher light output can be clearly seen up to $800 \mathrm{MeV}$ by choosing a wide neutron energy interval beyond $200 \mathrm{MeV}$.

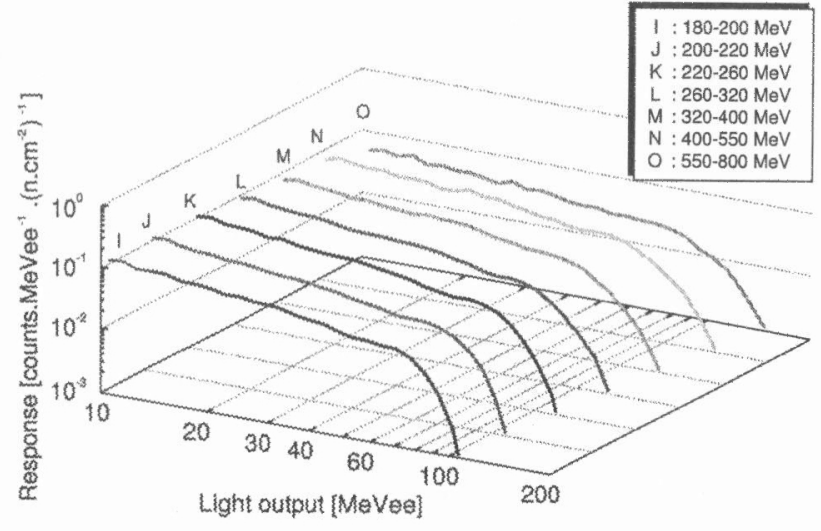

Figure 3. Measured response functions of the NE213 scintillator for $\{80-200,200-220,220-260,260-320,320-400,400-550$, and $550-$ $800 \mathrm{MeV}$ neutrons.

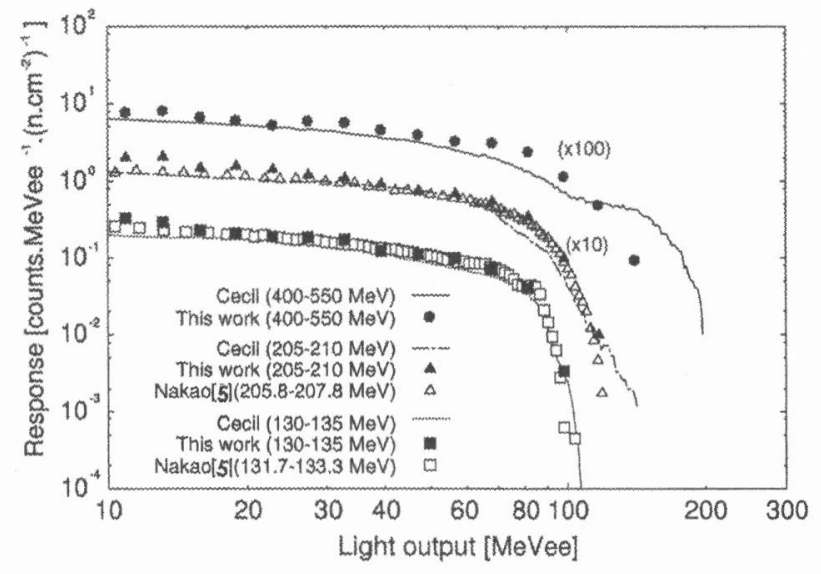

Figure 4. Measured response functions of the NE213 scintillator for $400-550,205-210$ and $130-135 \mathrm{MeV}$ neutrons ${ }^{6}$ compared with the CECIL calculation " and Nakao's data'

The event which induces such latge light output might be brought by the multiple collisions through the $\mathrm{C}(\mathrm{n}, x)$ reaction in the NE213 scintillator, and the resultant charged particles, such as Be, Li and alpha particle, have higher energy transfer from the incident neutron with increasing neutron energy.

The measured response functions to $130-135 \mathrm{MeV}$, $205-210 \mathrm{MeV}$ and $400-550 \mathrm{MeV}$ neutrons are compared with the results calculated by the CECLL code in Figure 4 . In the cases of $130-135 \mathrm{MeV}$ and $205-210 \mathrm{MeV}$ neutrons, two experimental tesults obtained by Sasaki et al. ${ }^{6}$ and by Nakao et al. ${ }^{5}$ are also compared and give very good agreement. The contribution to light output from the charged particles escaping from the detector is excluded in the CECIL calculation. For 130-135 MeV and 205-210 MeV neutrons, these experimental results and the CECIL calculations are in good agreement in absolute values, although a slight disagreement can be seen in the low light output region. For $400-450 \mathrm{MeV}$ neutrons, a large discrepancy in the high energy region can be seen. The difference of the upper end of light outputs between Sasaki's data and the CECIL calculation gradually increases with the neutron energy. This discrepancy is introduced by the inaccurate light output approximation used in the CECIL code.

This different increasing tendency of the light output with the incident neutron energies is clearly observed by comparison of the light output having the response value of $10^{-}$ (counts $\mathrm{MeVee}^{-3}\left(\mathrm{n} \mathrm{cm}^{-2}\right)^{-1}$, ee: electron-equivalent) between the experiment and the CECIL calculation as shown in Figure 5. The light outputs which were experimentally obtained monotonously increase with the neutron energy while some fluctuation can be observed in the CECL calculations. Both light outputs are in good agreement below $100 \mathrm{MeV}$, but the CECIL results indicate an overestimation from 100 to 200 


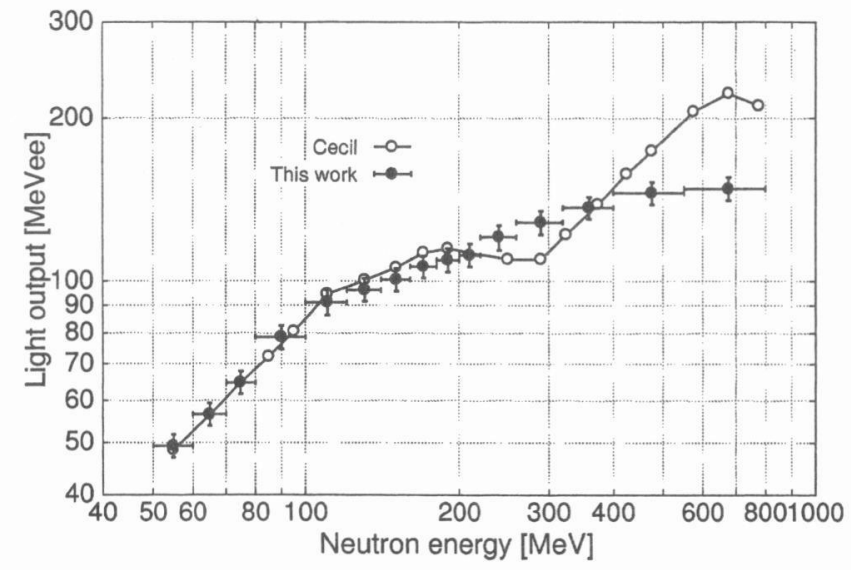

Figure 5. Comparison of the increasing tendency of the light output having the response value of $10^{-3}$ (counts $\mathrm{MeVee}^{-1}\left(\mathrm{n} \mathrm{cm}^{-2}\right)^{-1}$ ) for each incident neutron energy between the experiment and the CECIL calculation. $^{6}$

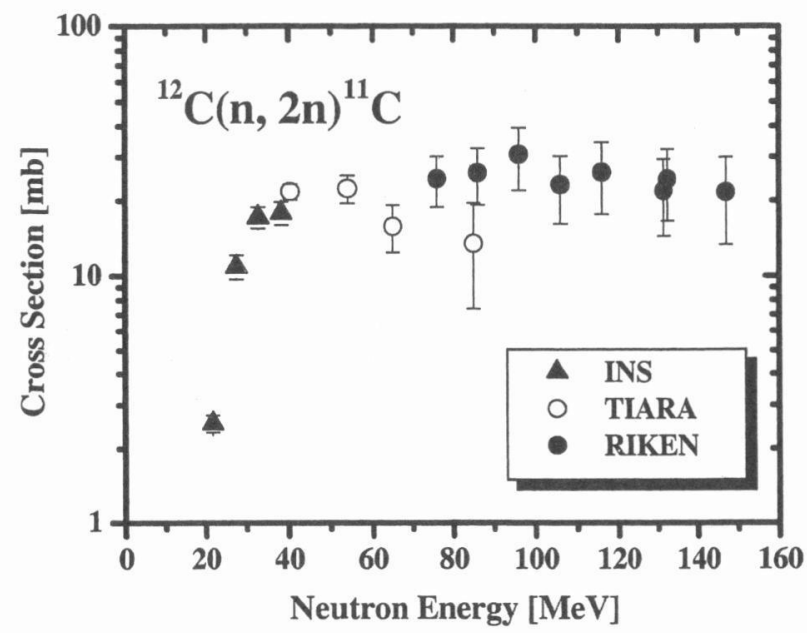

Figure 6. Measured ${ }^{12} \mathrm{C}(\mathrm{n}, 2 \mathrm{n})^{11} \mathrm{C}$ reaction cross section. ${ }^{9}$

$\mathrm{MeV}$, an underestimation from 200 to $350 \mathrm{MeV}$ and a large overestimation above $350 \mathrm{MeV}$. These discrepancies are introduced by the hypothesis of reaction cross sections being kept constant above $200 \mathrm{MeV}$. The CECIL code treats insufficient number of reaction channels and considers the light output only for protons and alpha particles. Therefore, it is not capable to simulate the pion production processes: e.g. $\mathrm{np} \rightarrow$ $\mathrm{d} \pi^{0}$ and $\mathrm{np} \rightarrow \mathrm{d} \pi^{-} \pi^{+}$.

In order to measure high-energy neutrons with higher efficiency and better energy resolution, a bigger $25.4 \mathrm{~cm}$ diam by $25.4 \mathrm{~cm}$ long NE213 scintillator is now in preparation for the experimental use. The response functions of this detector have also been measured preliminarily with HIMAC. ${ }^{8}$

II-2. Spallation Detectors of $\mathbf{C}$ and Bi. The spallation reactions of $\mathrm{C}$ and $\mathrm{Bi}$ induced by neutrons were used to detect high energy neutrons. The activation detectors are useful without a pulse pile-up problem in a burst-pulse field, but have generally low detection efficiencies. Large-volume activation detectors are therefore adopted in order to obtain high detection efficiencies. After neutron irradiations, the gamma rays from the activation detectors are measured with a high purity germanium (HPGe) detector.

The ${ }^{12} \mathrm{C}(\mathrm{n}, 2 \mathrm{n}){ }^{11} \mathrm{C}$ reaction has a threshold energy of about $20 \mathrm{MeV}$ and the cross section has recently been measured by Kim et al. ${ }^{9}$ up to $150 \mathrm{MeV}$ by using the p-Li quasi-monoenergetic neutron fields at the SF cyclotron facility at the Institute for Nuclear Study (INS), University of Tokyo, ${ }^{10}$ TIARA (Takasaki Institute of Advanced Radiation Research) AVF cyclotron facility ${ }^{11}$ and the RIKEN ring cyclotron facility. ${ }^{12}$ This reaction has an almost constant cross section of about 20

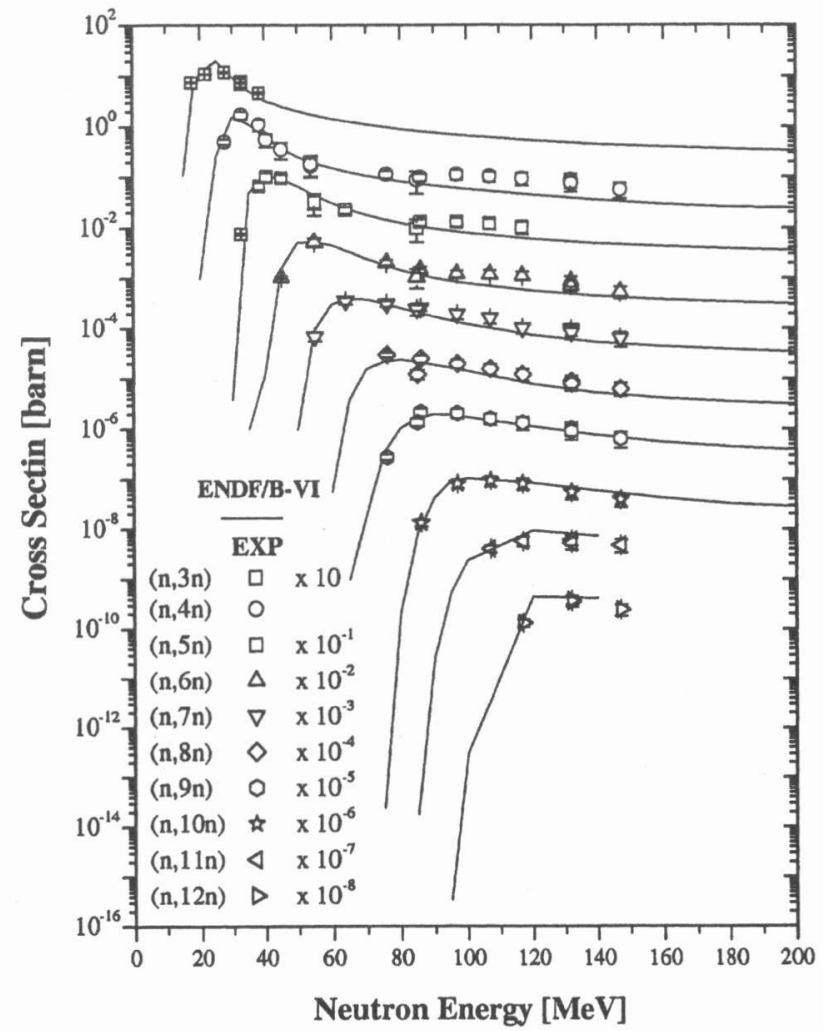

Figure 7. Measured ${ }^{209} \mathrm{Bi}(\mathrm{n}, x \mathrm{n})^{210-x} \mathrm{Bi}(x=3-12)$ reaction cross sections compared with the ENDF/B-VI high energy library data. ${ }^{9}$

$\mathrm{mb}$ above about $20 \mathrm{MeV}$ as shown in Figure 6. The half life of ${ }^{11} \mathrm{C}$ is about $20 \mathrm{~min}$, which makes a shortly-activated good neutron flux monitor for an energy above $20 \mathrm{MeV}$.

Bismuth detectors have been conventionally used for high energy neutron detection through the fission reaction, but our group found that the spallation reactions of ${ }^{209} \mathrm{Bi}(\mathrm{n}, x \mathrm{n}){ }^{210-x} \mathrm{Bi}$ $(x=3-12)$ are more useful for high-energy neutron spectrometry. The cross sections were also measured by Kim et al. ${ }^{9}$ in the energy range of 20 to $150 \mathrm{MeV}$ and the obtained data are in good agreement with the ENDF/B-VI high-energy library data ${ }^{13}$ calculated by Fukahori with the ALICE code, ${ }^{14}$ as can be seen in Figure 7. Their threshold energies regularly increase from $14 \mathrm{MeV}$ of ${ }^{209} \mathrm{Bi}(\mathrm{n}, 3 \mathrm{n})$ to $88 \mathrm{MeV}$ of ${ }^{209} \mathrm{Bi}(\mathrm{n}, 12 \mathrm{n})$ with an interval of $8 \mathrm{MeV}$ corresponding to the binding energy per nucleon. The half lives of ${ }^{200} \mathrm{Bi}$ to ${ }^{206} \mathrm{Bi}$ are between $36.4 \mathrm{~min}$ and 15.31 days. Table 1 shows the physical characteristics of ${ }^{12} \mathrm{C}(\mathrm{n}, 2 \mathrm{n}){ }^{11} \mathrm{C}$ and ${ }^{209} \mathrm{Bi}(\mathrm{n}, x \mathrm{n}){ }^{210-x} \mathrm{Bi}$ reactions.

The reaction rate, $R$, is connected to neutron cross section $\sigma(E)$ as follows:

$$
R=\int_{E_{\mathrm{th}}}^{E_{\max }} \sigma(E) \phi(E) d E
$$

where $\phi(E)=$ neutron fluence $\left[\mathrm{n} \mathrm{cm}^{-2} \mathrm{MeV}^{-1}\right]$,

$E_{\mathrm{th}}=$ threshold energy $[\mathrm{MeV}]$,

$E_{\max }=$ maximum energy of the monoenergetic peak neutrons $[\mathrm{MeV}]$.

II-3. Self-TOF Detector. Sasaki et al. ${ }^{15,16}$ developed the self-TOF detector for high energy neutron spectrometry behind a shield. The self-TOF detector consists of radiator detectors, a start counter and a stop counter of nine segments. A schematic drawing of the detector is given in Figure 8. The radiator, called RAD, is a stack of 20 thin plastic scintillators (NE102A) of $10 \mathrm{~cm} \times 10 \mathrm{~cm} \times 0.6 \mathrm{~cm}$. Each plastic scintillator is viewed by a $0.95 \mathrm{~cm}$ diam photo-multiplier (Hamamatsu R1635) through a light guide. The start counter, called START, is a plastic scintillator (NE102A) of $10 \mathrm{~cm} \times 10 \mathrm{~cm} \times$ $0.5 \mathrm{~cm}$ viewed by two $5.08 \mathrm{~cm}$ diam photo-multipliers (Hamamatsu R2083) from both sides through light guides. 
TABLE 1: Physical Characteristics of ${ }^{12} \mathrm{C}(\mathrm{n}, 2 \mathrm{n}){ }^{11} \mathrm{C}$ and ${ }^{209} \mathrm{Bi}(\mathrm{n}, \boldsymbol{x n})^{210-x} \mathrm{Bi}$ Reactions ${ }^{9}$

\begin{tabular}{|c|c|c|c|c|c|c|c|c|}
\hline \multirow{2}{*}{$\begin{array}{l}\text { Sample } \\
{ }^{12} \mathrm{C}\end{array}$} & \multirow{2}{*}{$\begin{array}{c}\begin{array}{c}\text { Production } \\
\text { Nuclei }\end{array} \\
{ }^{11} \mathrm{C}\end{array}$} & \multirow{2}{*}{$\begin{array}{l}\text { Nuclear Reaction } \\
{ }^{12} \mathrm{C}(\mathrm{n}, 2 \mathrm{n}){ }^{11} \mathrm{C}\end{array}$} & \multirow{2}{*}{$\begin{array}{l}\text { Q Value } \\
\text { /MeV } \\
-18.72\end{array}$} & \multirow{2}{*}{$\begin{array}{c}\begin{array}{c}\text { Threshold } \\
\text { Energy/MeV }\end{array} \\
20.4\end{array}$} & \multicolumn{2}{|c|}{$\begin{array}{l}\text { Half-Life } \\
\qquad\left(\mathrm{T}_{1 / 2}\right)\end{array}$} & \multirow{2}{*}{$\begin{array}{c}\text { Emission } \\
\text { Gamma Ray/keV } \\
511\end{array}$} & \multirow{2}{*}{$\begin{array}{c}\begin{array}{c}\text { Emission } \\
\text { Rate/\% }\end{array} \\
199.5\end{array}$} \\
\hline & & & & & 20.4 & $\min$ & & \\
\hline \multirow[t]{20}{*}{${ }^{209} \mathrm{Bi}$} & ${ }^{207} \mathrm{Bi}$ & ${ }^{209} \mathrm{Bi}(\mathrm{n}, 3 \mathrm{n}){ }^{207} \mathrm{Bi}$ & -14.35 & 14.42 & 38 & $\mathrm{y}$ & 569.7 & 97.8 \\
\hline & & & & & & & 1063.6 & 74.9 \\
\hline & ${ }^{206} \mathrm{Bi}$ & ${ }^{209} \mathrm{Bi}(\mathrm{n}, 4 \mathrm{n})^{206} \mathrm{Bi}$ & -22.44 & 22.55 & 6.243 & & 803.1 & 98.9 \\
\hline & & & & & & & 881.1 & 66.2 \\
\hline & ${ }^{205} \mathrm{Bi}$ & ${ }^{209} \mathrm{Bi}(\mathrm{n}, 5 \mathrm{n}){ }^{205} \mathrm{Bi}$ & -29.48 & 29.62 & 15.31 & d & 703.4 & 31 \\
\hline & & & & & & & 1763.4 & 32.4 \\
\hline & ${ }^{204} \mathrm{Bi}$ & ${ }^{209} \mathrm{Bi}(\mathrm{n}, 6 \mathrm{n}){ }^{204} \mathrm{Bi}$ & -37.94 & 38.13 & 11.30 & $\mathrm{~h}$ & 899.2 & 99.2 \\
\hline & & & & & & & 983.9 & 58.4 \\
\hline & ${ }^{203} \mathrm{Bi}$ & ${ }^{209} \mathrm{Bi}(\mathrm{n}, 7 \mathrm{n})^{203} \mathrm{Bi}$ & -45.15 & 45.37 & 11.76 & $\mathrm{~h}$ & 820.5 & 29.7 \\
\hline & & & & & & & 825.2 & 14.6 \\
\hline & ${ }^{202} \mathrm{Bi}$ & ${ }^{209} \mathrm{Bi}(\mathrm{n}, 8 \mathrm{n}){ }^{202} \mathrm{Bi}$ & -53.98 & 54.24 & 1.670 & & 422.1 & 83.8 \\
\hline & & & & & & & 960.6 & 99.4 \\
\hline & ${ }^{201} \mathrm{Bi}$ & ${ }^{209} \mathrm{Bi}(\mathrm{n}, 9 \mathrm{n}){ }^{201} \mathrm{Bi}$ & -61.39 & 61.69 & 1.770 & & 628.2 & 24.5 \\
\hline & & & & & & & 935.7 & 11.3 \\
\hline & ${ }^{200} \mathrm{Bi}$ & ${ }^{209} \mathrm{Bi}(\mathrm{n}, 10 \mathrm{n}){ }^{200} \mathrm{Bi}$ & -70.56 & 70.89 & 36.40 & $\min$ & 462.2 & 98 \\
\hline & & & & & & & 1026.2 & 100 \\
\hline & ${ }^{199} \mathrm{Bi}$ & ${ }^{209} \mathrm{Bi}(\mathrm{n}, 11 \mathrm{n}){ }^{199} \mathrm{Bi}$ & -78.1 & 78.47 & 24.7 & $\min$ & 425 & 22 \\
\hline & & & & & & & 841 & 11 \\
\hline & ${ }^{198} \mathrm{Bi}$ & ${ }^{209} \mathrm{Bi}(\mathrm{n}, 12 \mathrm{n}){ }^{198} \mathrm{Bi}$ & -87.52 & 87.94 & 11.1 & $\min$ & 562.4 & 79 \\
\hline & & & & & & & 1063.4 & 100 \\
\hline
\end{tabular}

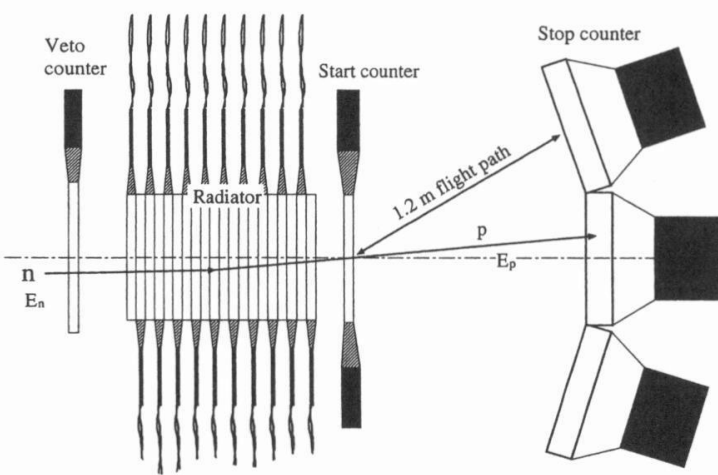

Figure 8. Schematic drawing of the self-TOF detector. ${ }^{15,16}$

The stop counter, called STOP, is designed to cover an area of $60 \mathrm{~cm} \times 60 \mathrm{~cm}$ and is segmented into nine plastic scintillators (NE102A) of $20 \mathrm{~cm} \times 20 \mathrm{~cm} \times 2 \mathrm{~cm}$, each of which is viewed by a $12.7 \mathrm{~cm}$ diam photo-multiplier (Hamamatsu R1259) through a light guide. The distance between the start and stop counters is adjustable, and is chosen to be $1.2 \mathrm{~m}$ for a usual measurement.

An in-coming neutron produces charged particles in twenty radiators, and then the charged particles emitted in the forward direction reach any one of nine stop counters through the start counter. The energy of the charged particle is determined by using the TOF (Time-of-Flight) method between the start and stop counters. In this detector, only proton events from $\mathrm{H}(\mathrm{n}, \mathrm{p})$ and $C(n, p)$ reactions are selected to obtain the detector response function. The neutron energy spectrum can be obtained from the measured proton energy spectrum using an unfolding method with the response functions.

The response functions of the self-TOF detector for highenergy neutrons up to $800 \mathrm{MeV}$ were measured at HIMAC of NIRS. Figure 9 shows the experimental setup at HIMAC. The neutrons were produced by bombarding $400 \mathrm{MeV} /$ nucleon ${ }^{12} \mathrm{C}$ ion and $800 \mathrm{MeV} /$ nucleon ${ }^{28} \mathrm{Si}$ ion beams with an $\mathrm{RF}$ structure on thick (stopping-length) carbon target. The target size was $10 \mathrm{~cm} \times 10 \mathrm{~cm}$ and $20 \mathrm{~cm}$ thick for $400 \mathrm{MeV} /$ nucleon ${ }^{12} \mathrm{C}$, and $10 \mathrm{~cm} \times 10 \mathrm{~cm}$ and $23 \mathrm{~cm}$ thick for $800 \mathrm{MeV} /$ nucleon ${ }^{28} \mathrm{Si}$ ion. The thin NE102A plastic scintillator as a beam pickup detector, called PICK, (30 $\mathrm{mm}$ diam and $0.5 \mathrm{~mm}$ thick) was placed just behind the end window of beam line. This PICK

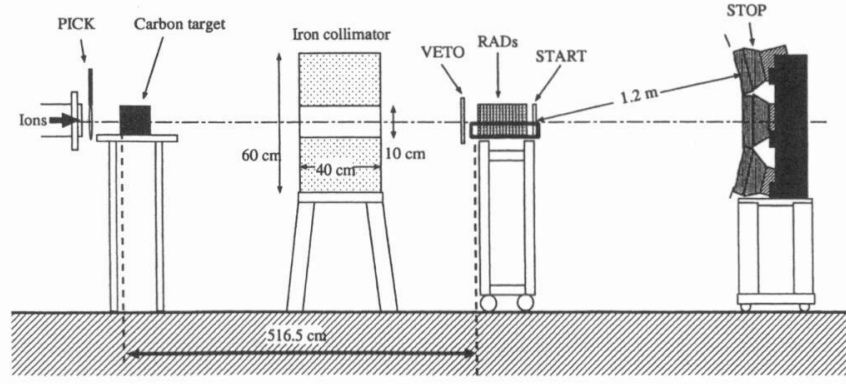

Figure 9. Experimental setup at the HIMAC. ${ }^{16}$

detector was used to count the number of projectiles and also as a start signal for conventional neutron TOF measurement. The carbon target was placed just behind the PICK detector.

The self-TOF detector was placed $516.5 \mathrm{~cm}$ downstream from the target front face. It is already known that this radiation field has a lot of charged particles generated by fragmentation reactions, ${ }^{17}$ especially in the forward direction. Accordingly the detector was placed at 15 degrees to the beam line to avoid a large amount of charged fragment particles. Furthermore, the iron collimator of $60 \mathrm{~cm} \times 60 \mathrm{~cm}$ and $40 \mathrm{~cm}$ thick with a hole of $10 \mathrm{~cm} \times 10 \mathrm{~cm}$ was set in front of the selfTOF detector to decrease the accidental signals which were induced by the incidence of charged fragment particles on the STOP counters, and also to inject neutrons almost normally into the detector. The veto counter, called VETO, $(150 \mathrm{~mm} \times$ $150 \mathrm{~mm}$ and $5 \mathrm{~mm}$ thick NE102A plastic scintillator), was placed in front of the radiator to reject charged particle events from neutron events.

The data were analyzed as follows: (i)Select only the proton events generated in the radiators by using the light output data of the veto counter and the most downstream radiator, (ii)Select only the proton event from the two dimensional plot of time-of-flight versus light output of stop counter, and (iii)Convert TOF spectrum into proton energy spectrum.

When all of the events selected are the proton events, the TOF, $T_{\mathrm{p}}(x)$ appeared at the channel $x$ is given by the following equation,

$$
T_{\mathrm{p}}(x)=\left(x-x_{0}\right) \cdot \Delta T
$$


where $x_{0}$ is the channel which corresponds to the time when a proton passes through the start counter, $\Delta T$ is the time per channel in ns which is determined by a time calibrator module. Then, the proton energy, $E_{\mathrm{p}}(x)$, is determined by the following equation using the relativistic kinematics,

$$
\begin{gathered}
E_{\mathrm{p}}(x)=M_{0} c^{2}\left(\frac{1}{\sqrt{1-\beta^{2}}}-1\right) \\
\beta=\frac{V_{\mathrm{p}}(x)}{c}=\frac{L}{c \cdot T_{\mathrm{p}}(x)}
\end{gathered}
$$

where $M_{0}$ is the rest mass of a proton, $V_{\mathrm{p}}$ is the proton velocity, $c$ is the light velocity and $L$ is the flight path of $1.2 \mathrm{~m}$.

The correction for proton energy loss in the radiator detectors is not necessary to be considered because the obtained proton energy spectra are used as response functions of the whole detector system. The incident neutron energy from the source is determined by the TOF between the beam pickup detector and the start counter. By repeating the analysis mentioned above with changing the ROI of the neutron energy, the neutron response function matrices can be obtained between $60 \mathrm{MeV}$ and $800 \mathrm{MeV}$.

The measured responses were compared with those calculated using the LCS (LAHET Code System). ${ }^{18}$ As examples, Figures 10 and 11 show the responses for 100 to $120 \mathrm{MeV}$ neutrons and for 450 to $500 \mathrm{MeV}$ neutrons, respectively, with the results calculated by the LCS. The LCS results considerably overestimate the measured results for higher neutron energy. By using the measured response matrices, the neutron detection efficiency of the detector was obtained as shown in Figure 12 (solid line) by comparing with the detection efficiency for $10 \mathrm{MeV}$ ee threshold of the NE213 organic liquid scintillator ${ }^{5}$ calculated by the CECIL code (broken line). ${ }^{7}$ This detector has a good efficiency for high-energy neutrons above

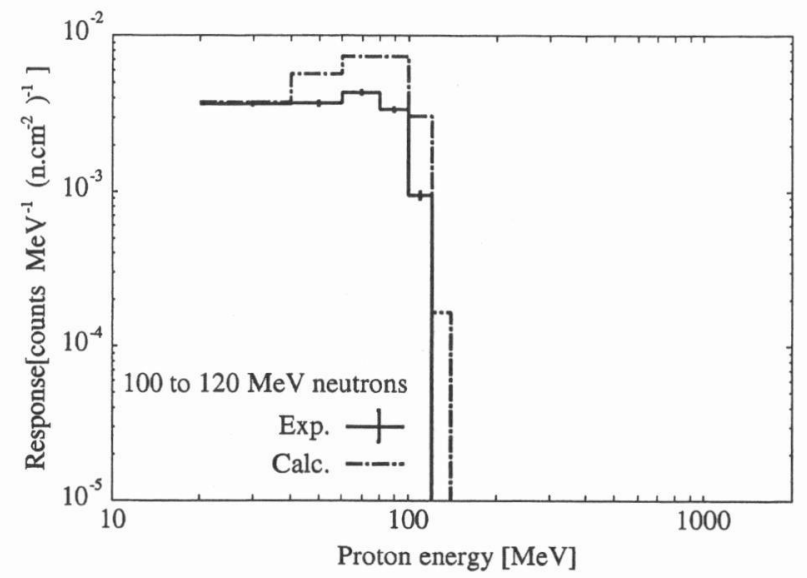

Figure 10. Comparison of measured and calculated response functions for 100 to $120 \mathrm{MeV}$ neutrons. ${ }^{16}$

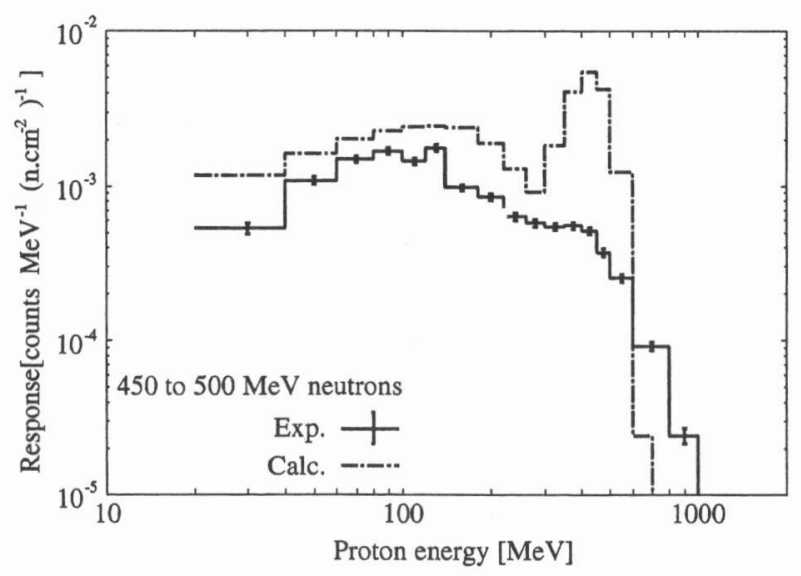

Figure 11. Comparison of measured and calculated response functions for 450 to $500 \mathrm{MeV}$ neutrons. ${ }^{16}$ about $100 \mathrm{MeV}$.

This detector can give the neutron energy spectrum by using the FERDO-U ${ }^{19}$ or FORIST ${ }^{20}$ unfolding code combined with the measured response functions, and is useful for high energy neutron spectrometry because of the almost constant efficiency for neutrons above $100 \mathrm{MeV}$.

\section{Neutron Detectors for Use in a Mixed Field of Charged Particles and Neutrons}

III-1. Phoswich Detector of NE115 and NE213. In the phoswich detector developed by Takada et al., ${ }^{21}$ the two scintillators are optically coupled to a single photomultiplier tube (Hamamatsu H1949). A cross-sectional view is shown in Figure13. The liquid scintillator is surrounded by a thin slow plastic scintillator (NE115, $5 \mathrm{~mm}$ thick) with a low sensitivity to neutral particles. The inner detector is a liquid organic scintillator (NE213, $58.5 \mathrm{~mm}$ diam and $58 \mathrm{~mm}$ length which corresponds to the range of $70 \mathrm{MeV}$ proton) contained in the glass cell and has much greater sensitivity to neutrons. Charged particles are detected by both scintillators. The light output pulse in the NE115 from charged particles has a long characteristic time constant of about $225 \mathrm{~ns}$, whereas the light output pulse in the NE213 by recoil protons from energetic neutrons has a time constant of about $30 \mathrm{~ns}$ and that by Compton electrons from gamma rays is about $3.7 \mathrm{~ns}$. These differences in the light-decay time constant make it possible to separate pulses of the three different particle species.

Protons, neutrons and gamma rays are detected separately by use of a pulse-shape discrimination technique based on the standard CAMAC charge integration ADCs. The charge integration of the signal is carried out during the time period specified by a gate pulse (total-gate, slow-gate). The total and slow components can be analyzed by a total gate pulse adjusted at

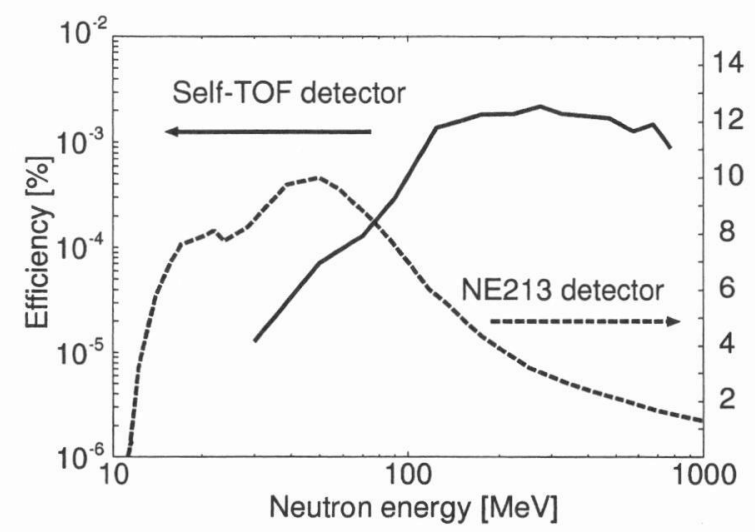

Figure 12. Neutron detection efficiencies of the self-TOF detector (solid line) and NE213 organic liquid scintillator (broken line). ${ }^{16}$

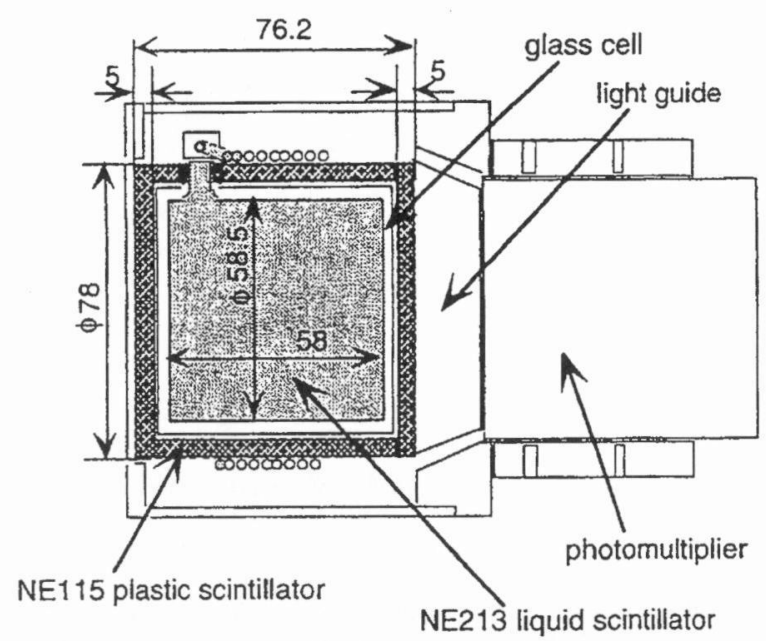

Figure 13. Diagram of the phoswich detector. ${ }^{21,22}$ 
the peak of the signal and by a wider delayed slow gate set at the long tail of the signal, respectively.

Experiments were carried out at two cyclotron facilities: the ring cyclotron facility at RIKEN and the AVF cyclotron facility at NIRS. They produced mixed fields of neutrons and protons by bombarding $135 \mathrm{MeV}$ protons on a $10 \mathrm{~mm}$ thick $\mathrm{Li}$ target at RIKEN and by bombarding $70 \mathrm{MeV}$ protons on a 2 $\mathrm{mm}$ thick Be target at NIRS. The detector was placed at 5-10 $\mathrm{m}$ downstream from the target at $30-45$ degrees to the proton beam axis as shown in Figure14. A thin plastic scintillation detector (NE102A) was positioned in front of the phoswich detector. This thin detector was used to discriminate between neutrons and protons for confirming the particle identification ability of the phoswich detector.

A two-dimensional spectrum of the light output of the slow component versus the total component measured with the phoswich detector at NIRS is presented in Figure 15 as an example. In this figure, there are seven distinct groups, labeled "A" through "G." The component "A" comes from the electrons scattered by gamma rays. The four components " $B$ " through "E" containing small fractions of slow components are due to neutrons, where the components " $B$ " and " $C$ " correspond to recoiled protons produced by elastic collisions of neutrons with hydrogen in the liquid scintillator. The component " $D$ " represents deuterons attributed to the ${ }^{12} \mathrm{C}(\mathrm{n}, \mathrm{d})$ reaction, and the component "E" represents alpha particles produced in the ${ }^{12} \mathrm{C}(\mathrm{n}, \alpha)$ and ${ }^{12} \mathrm{C}(\mathrm{n}, \mathrm{n}, 3 \alpha)$ reactions. Some fractions of component " $\mathrm{B}$ " is due to protons that lost some energy in the NE213 and then escaped from the NE213. The component " $\mathrm{C}$ " corresponds to protons that deposited all of their energy in the NE213. The components "F" and "G"

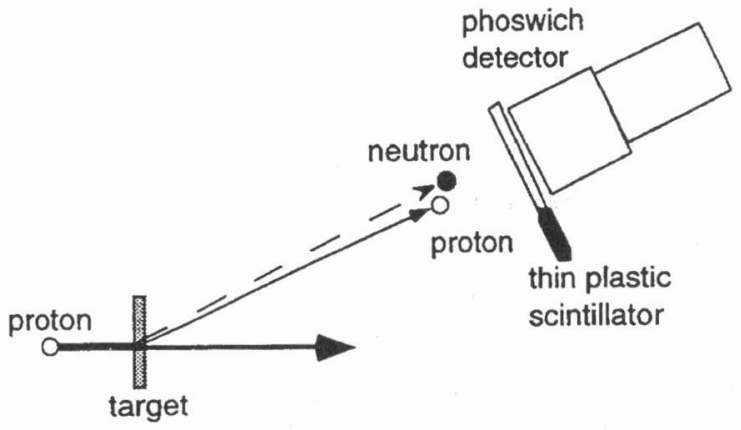

Figure 14. Schematic view of the experimental set up in a protonmixed field at NIRS and RIKEN..$^{21,22}$

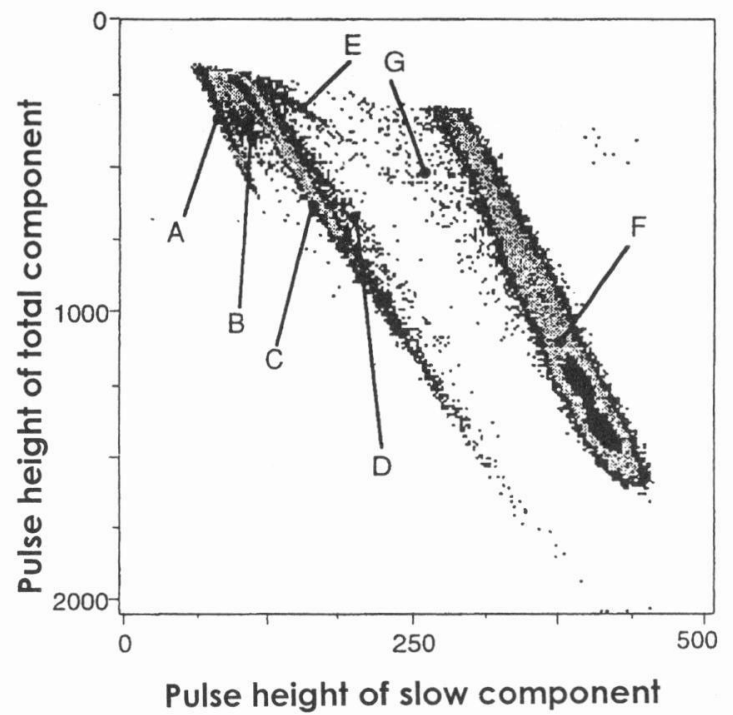

Figure 15. A two-dimensional pulse height spectrum of the slow component versus the total component measured with the phoswich detector at the NIRS. The component "A" shows gamma-ray events, the components "B" through "E" show neutron events and the components " $F$ " and " $G$ " show proton events. ${ }^{21,22}$ doubtless contain external protons reaching the NE213 through the NE115 scintillator and the glass cell. The events where the external protons stopped in the NE115 are not seen in this figure because of the detection logic.

All events in the two-dimensional plot of Figure 15 were projected onto the total component axis to obtain individual spectra of gamma-ray, neutron and proton events, as shown in Figure 16. We can see in these spectra that the discrimination between gamma rays, neutrons and protons is excellent, and that the proton peaks are clearly identifiable. This proton peak corresponds to the elastically scattered one toward the detector from the target having about $58 \mathrm{MeV}$ energy.

The response functions of this type phoswich detector of larger size $(15 \mathrm{~mm}$ thick NE115 and $133 \mathrm{~mm}$ diam by $133 \mathrm{~mm}$ long NE213) were also measured for neutrons up to $130 \mathrm{MeV}$ at RIKEN using a white neutron source of $135 \mathrm{MeV}$ proton on thick Be and $\mathrm{C}$ targets, coupled with the neutron TOF technique. By using the measured response functions, the neutron energy spectrum can be obtained separately from the gammaray and proton energy spectra by unfolding with the FERDO-U code,$^{19}$ as shown in Figure 17.22 The proton has a monoenergetic peak at around $57 \mathrm{MeV}$ which corresponds to the elastically scattered proton energy of $58 \mathrm{MeV}$. The neutron spectrum has a continuous energy distribution extended to about $70 \mathrm{MeV}$.

III-2. Anti-coincidence System of $\Delta E$ and $E$ Counters. The $\Delta E$ and $E$ counter telescope with the anti-coincidence detection system is widely used for particle identification. We used this detection system to measure neutron energy spectra

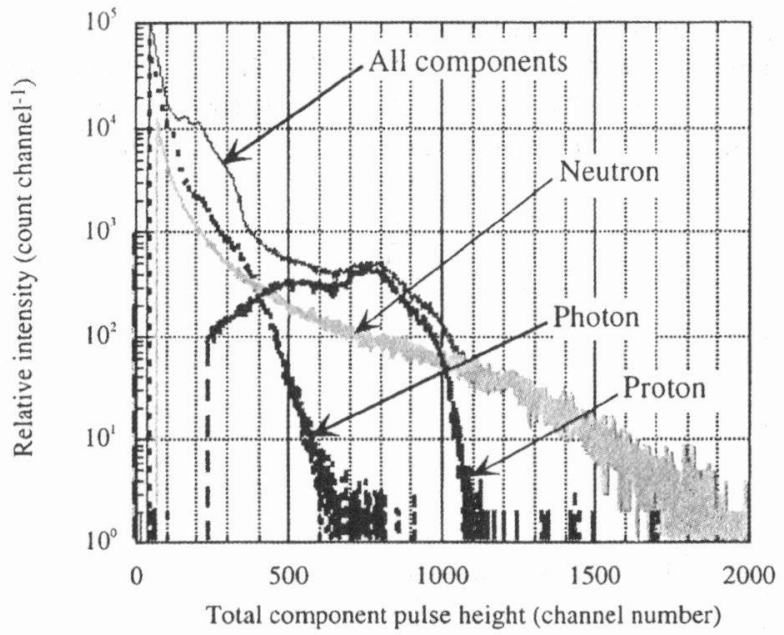

Figure 16. The light output spectra projected on the total component axis obtained by discriminating between gamma-ray, neutron and proton events in Figure 15. ${ }^{21,22}$

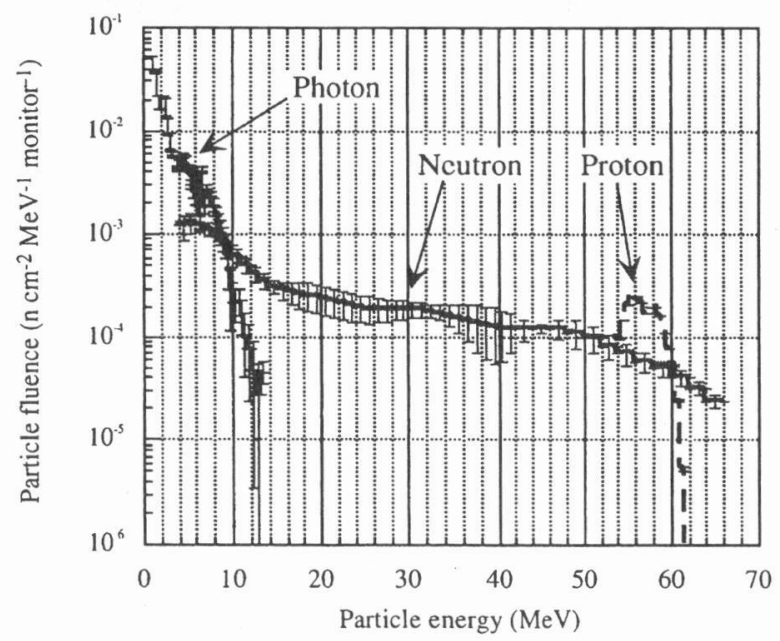

Figure 17. Neutron, proton and gamma-ray energy spectra ${ }^{21,22}$ obtained by the FERDO-U unfolding code. ${ }^{19}$ 
produced from the high energy heavy ions at HIMAC. ${ }^{23}$

The NE213 liquid scintillator $(12.7 \mathrm{~cm}$ diam by $12.7 \mathrm{~cm}$ thick) coupled with the R4144 photomultiplier connected to E1458 base (Hamamatsu) were used for the neutron detector ( $E$ counter). The NE102A plastic scintillator $(15 \mathrm{~cm}$ by $15 \mathrm{~cm}$ square and $0.5 \mathrm{~cm}$ thick), coupled with the H1949 photomultiplier and base (Hamamatsu), were used for a $\Delta E$ counter and were placed in front of the $E$ counter to discriminate charged particles from uncharged particles, neutrons and photons. The total and slow gate timings for the neutron-photon discrimination with respect to the analog signals were shown in Figure 18.

To eliminate charged particles, we used two-dimensional $\Delta E-E$ graphical plots (Figure 19). As neutrons and gamma rays do not scintillate the $\Delta E$ counter, the neutron and gammaray events could be selected from the charged particle events as seen in Figure 19. After this discrimination, the neutron and gamma-ray events were separated by using two-dimensional, total-slow pulse-height graphical plots (Figure 19). In this discrimination, the pulse shapes from high energy neutron events in which recoil protons escape from the $E$ counter are close to those from gamma-ray events, and these events were eliminated from the neutron events.

The neutron energy spectrum can also be obtained by unfolding with the FERDO-U code coupled with the pre-determined response functions. In space, the charged particles and neutrons enter into the detector from all directions. Then the $\Delta E$ counter must fully cover the $E$ counter for neutron identification. As a result, the $\Delta E-E$ counter telescope inevitably becomes rather big.

III-3. Bonner Sphere with ${ }^{3} \mathrm{He}$ Counter. The Bonner sphere (Bonner ball) fabricated by the National Space Development Agency (NASDA) and Mitsubishi Research Institute Inc. (MRI) consists of six ${ }^{3} \mathrm{He}$ proportional counters covered with a neutron moderator of different thickness. ${ }^{24}$ The ${ }^{3} \mathrm{He}$ proportional counters at the center of the six sensors are identical, $5.08 \mathrm{~cm}$ diam spheres and filled with $5 \mathrm{~atm}{ }^{3} \mathrm{He}$ gas. Each counter is covered by a polyethylene moderator of different thickness $(1.5 \mathrm{~cm}, 3 \mathrm{~cm}, 5 \mathrm{~cm}$, and $9 \mathrm{~cm})$, and neutron energy spectra can be obtained through the unfolding technique using the difference in the neutron sensitivity function

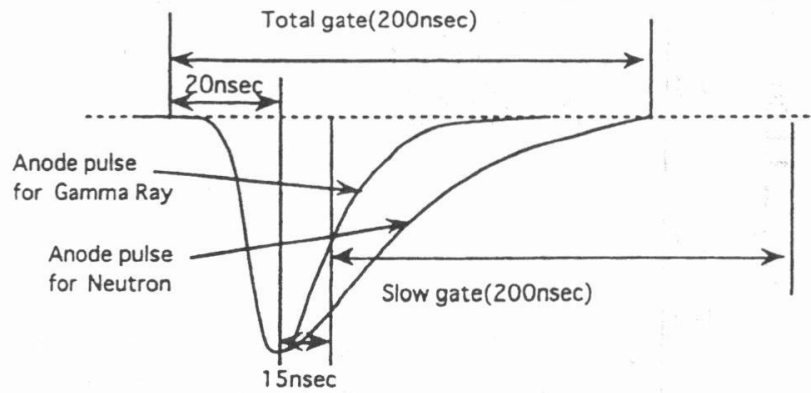

Figure 18. The gate timing of the total and slow pulse heights.

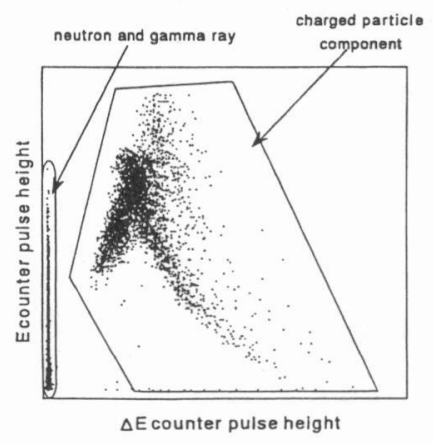

(a)

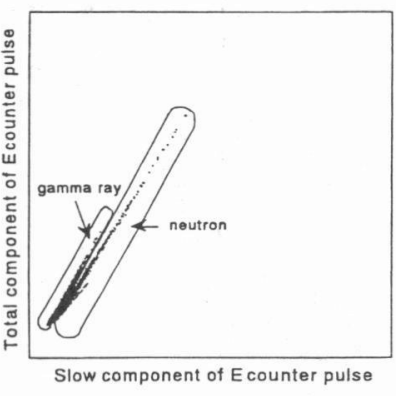

(b)
Figure 19. Two-dimensional plots of $E$ and $\Delta E$ counters for (a) the pulse heights and (b) the total and slow pulse heights. ${ }^{23}$ (energy response function) obtained for the various thicknesses of polyethylene moderator. This Bonner sphere is the same one as that developed by Uwamino et al., ${ }^{25}$ but adjusted for use in space. Figure 20 shows the cross sectional view of sensors. Sensor 2 and sensor 3 are covered with $1 \mathrm{~mm}$-thick gadolinium to block thermal neutrons.

The response functions of the Bonner ball were calculated in the neutron energy range of $0.025 \mathrm{eV}$ to $100 \mathrm{MeV}$ by using the MCNP-4B Monte Carlo code ${ }^{26}$ with several cross section data libraries, and the calculation accuracy is checked by the monoenergetic neutron measurements at the Fast Neutron Laboratory (FNL) of Tohoku University. The neutron energy spectrum can be obtained by unfolding with the SAND-2 $\operatorname{code}^{27}$ under a certain initial guess spectrum.

The Bonner ball detector was mounted in the SPACEHAB module in the space shuttle cargo bay, and the neutron energy spectrum in the shuttle was measured on January 24 to 28 , 1998 in S/MM-08 (STS-89). Measured data were monitored through downlink at the NASA Johnson Space Center Mission Control Center and transmitted for analysis with orbital data to Tsukuba Space Center, NASDA. The output pulse-height distribution obtained from each Bonner ball presented a sharp thermal neutron peak, and neutrons could be clearly distinguished from protons and other particles. Figure 21 compares the predicted pulse-height distribution (worst case) and the measured output pulse-height distribution. Figure 22 gives the three data of the neutron energy spectra obtained at the equatorial region, polar region and SAA (South Atlantic Anomaly)
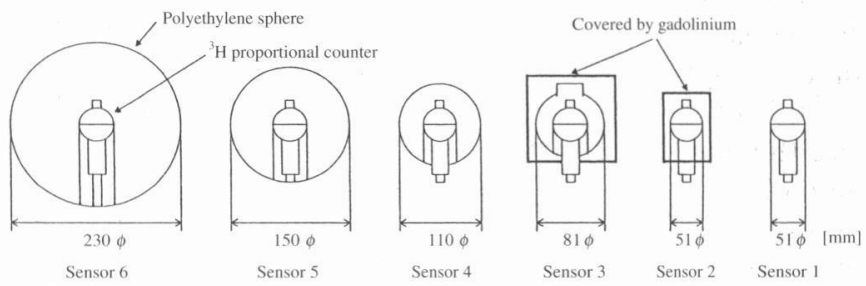

Figure 20. Cross sectional view of each sensor. ${ }^{24}$
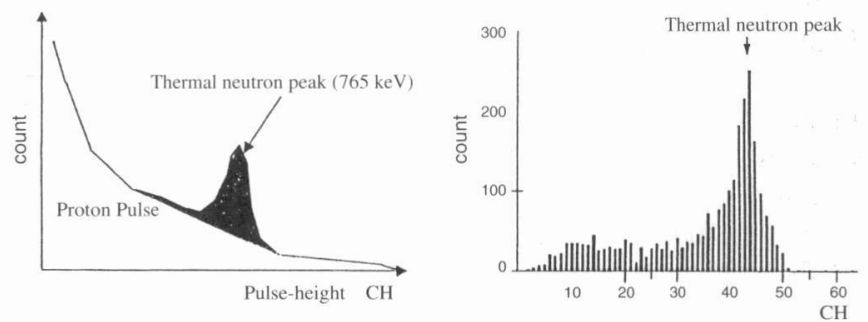

Figure 21. Output pulse height distribution. (a) Predicted pulseheight distribution. (b) Measured output pulse-height. ${ }^{24}$

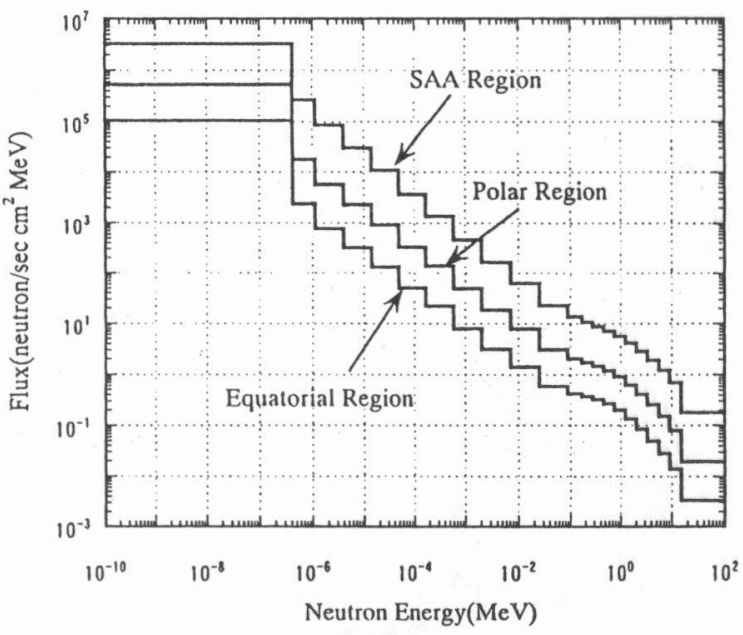

Figure 22. Neutron energy spectra inside shuttle at three regions. ${ }^{24}$ SAA means the South Atlantic Anomaly. 
region under the initial guess spectrum that the neutron energy spectrum $\phi(E)$ is proportional to $1 / E .^{24}$ These three spectra are close together in their shapes, but the magnitude at SAA is about 100 and 1000 times higher than the polar region and the equatorial region, respectively.

III-4. Bonner Sphere with ${ }^{6} \mathrm{Li}$ and ${ }^{7} \mathrm{Li}$ Glass Scintillators. Two types of the Bonner sphere with a pair of ${ }^{6} \mathrm{Li}$ and ${ }^{7} \mathrm{Li}$ glass scintillators were developed. One type uses many pairs of ${ }^{6} \mathrm{Li}$ and ${ }^{7} \mathrm{Li}$ glass scintillators inserted into one polyethylene moderator which has been developed by Yamaguchi et al. ${ }^{28}$ and the other uses one pair of ${ }^{6} \mathrm{Li}$ and ${ }^{7} \mathrm{Li}$ glass scintillator mounted into several polyethylene moderators of different thickness which has been developed by Taniguchi et al. ${ }^{29}$

An illustration of the former detector is shown in Figure 23. The detector consists of a spherical polyethylene moderator with a diameter of $30 \mathrm{~cm}$ and 19 pairs of ${ }^{6} \mathrm{Li}$ - and ${ }^{7} \mathrm{Li}$-glass scintillators (10 $\mathrm{mm}$ diam with $2 \mathrm{~mm}$ thickness) arranged along the orthogonal $x, y$, and $z$ axes of the sphere. Each scintillator is coupled to a thin photomultiplier tube (Hamamatsu $\mathrm{R} 5600 \mathrm{P}$ ) using an acrylic light-guide with silicon grease. The scintillator, the light-guide and the photomultiplier are covered with a black light-shield case which are made of a similar material to polyethylene and do not obstruct the thermal neutron flux in the spherical moderator.

The different response between the ${ }^{6} \mathrm{Li}$ and ${ }^{7} \mathrm{Li}$ glass scintillators enables to distinguish neutrons from other radiation. The ${ }^{6} \mathrm{Li}$-glass scintillators are sensitive to both thermal neutrons and the other radiation. The ${ }^{7} \mathrm{Li}$ glass scintillators are sensitive only to radiation except for thermal neutrons. The thermal neutron flux at the scintillator pair can be obtained by subtracting the number of events detected by the ${ }^{7} \mathrm{Li}$-glass scintillator from that by ${ }^{6} \mathrm{Li}$-glass one. The dose equivalent and the energy spectrum can be obtained with a proper unfolding process. The present counter allows to obtain the information with only one measurement by using the position information of the thermal neutron flux in the sphere.

Three monoenergetic neutron beams with energies of 165 $\mathrm{keV}, 565 \mathrm{keV}$ and $5 \mathrm{MeV}$ were generated by a CockcroftWalton accelerator at the Electro-Technical Laboratory. ${ }^{28}$ Figure 24 presents the response of the detector obtained by the axial scintillator pairs at the three angles for the respective monoenergetic neutron. The points stand for the number of the neutron detection events obtained by each scintillator pair. The line shows the thermal neutron flux distribution expected from the experimental points. First, comparing the responses of the $x$-axial scintillator pairs at an angle of $0^{\circ}$ obtained at different energies of the beams, the thermal neutron flux distribution is extended to the center of the spherical moderator with increase of the neutron energy. The responses of the $y$ - and $z$ -

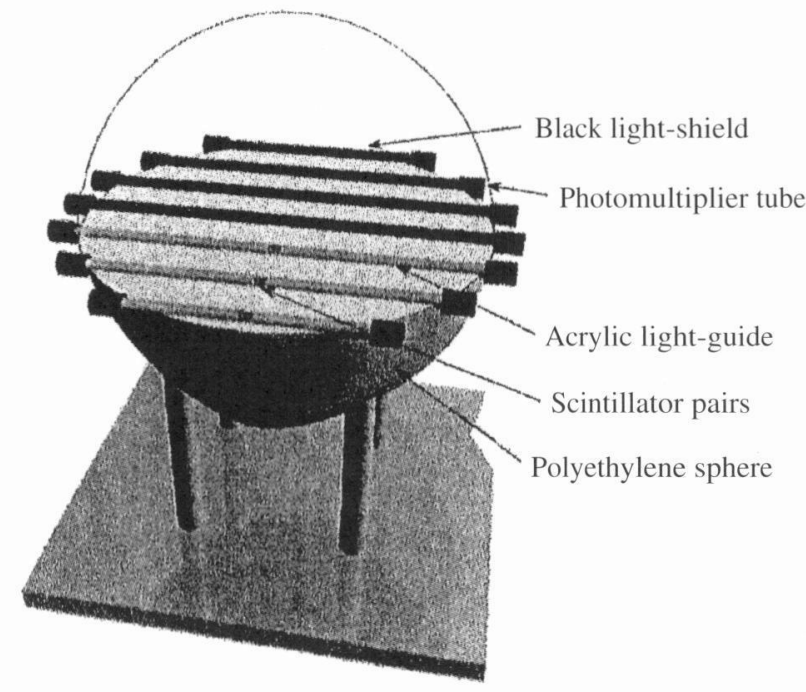

Figure 23. A schematic drawing of the Bonner sphere. ${ }^{28}$ axial scintillator pairs show a good symmetry about the center of the sphere. The number of the neutron detection events increases with neutron energy similar to that of the $x$-axial scintillator pairs. These responses of the detector express the characteristics of the neutron mean free pass in polyethylene. Second, comparing the responses of the $x$-axial scintillator pairs at different angles, the response varied reasonably with the change of the incident angle. The detector shows an excellent response against the different energy and the different direction of the neutrons.

The results demonstrate that the present detector allows to obtain much information about incident neutrons. This ability of the detector, however, was checked only for the monoenergetic neutron beam and the point-like neutron source, not checked in isotropic and proton existing fields. For practical use of the detector in the space environment, characterization of the detector in such fields is necessary.

The latter detector uses a pair of $2.54 \mathrm{~cm}$ diam $\times 2.54 \mathrm{~cm}$ long NE912 (7.7 wt $\%$ of 95\% ${ }^{6} \mathrm{Li}$-enriched lithium doped) and NE913 (8.3 wt \% of $99.99 \%{ }^{7}$ Li-enriched lithium doped) glass scintillators. Each of these two scintillators is coupled with a photo-multiplier (Hamamatsu R1924) mounted out of the moderator through the acrylic light-guide. The spherical moderators are made of polyethylene and their thicknesses are $1.5,3.0,5.0$, and $9.0 \mathrm{~cm}$. A schematic view of the spectrometer is shown in Figure 25. The response functions to neutrons from $10^{-9} \mathrm{MeV}$ up to $200 \mathrm{MeV}$ were calculated ${ }^{29}$ by the MCNPX Monte Carlo calculation ${ }^{30}$ with the ENDF/B-VI neutron cross section data library. ${ }^{13}$

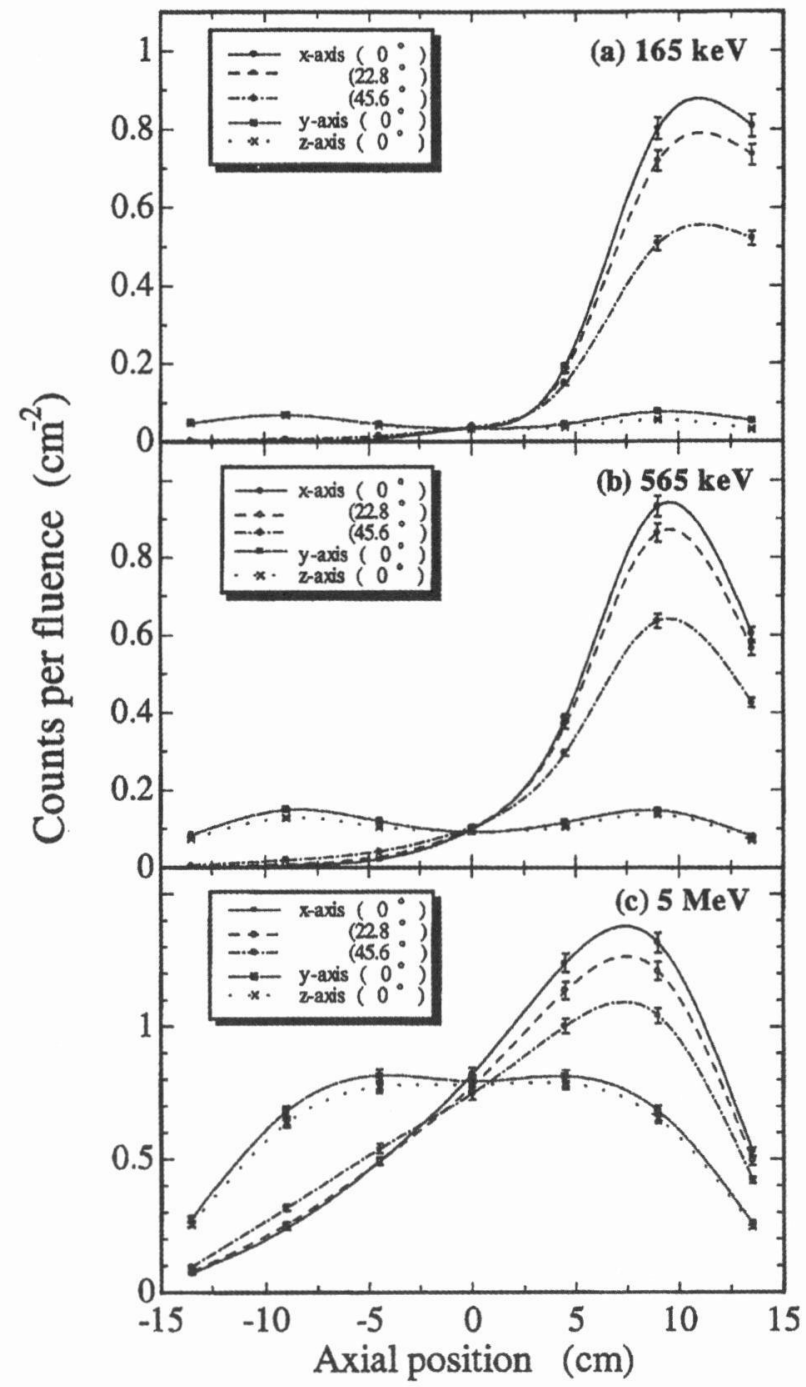

Figure 24. Experimentally obtained response of the axial scintillator pairs for monoenergetic neutron beams of (a) $165 \mathrm{keV}$, (b) $565 \mathrm{keV}$ and (c) $5 \mathrm{MeV}$ from angles of $0^{\circ}, 22.8^{\circ}$ and $45.6^{\circ} .^{28}$ 


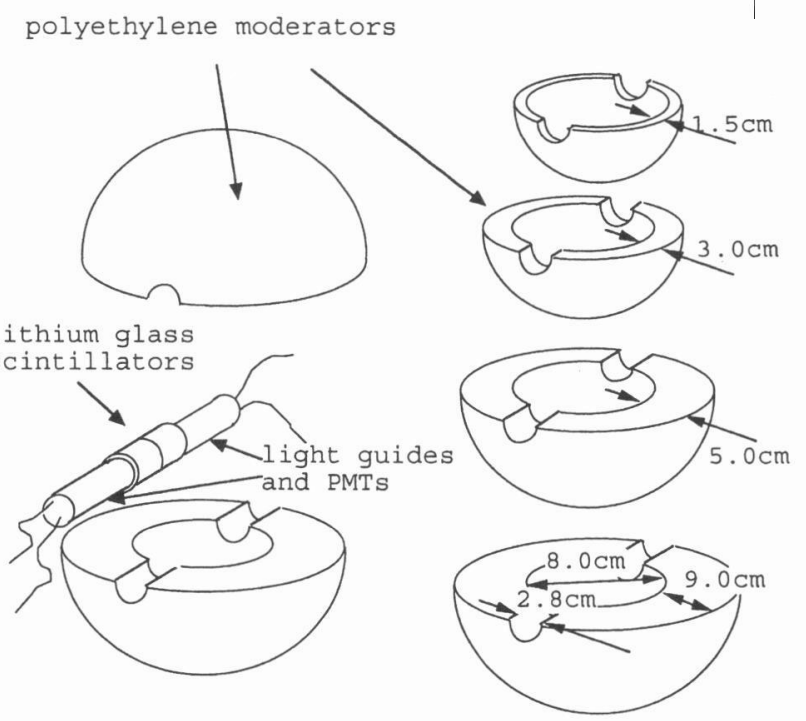

Figure 25. Schematic view of the multi-moderator spectrometer with a pair of lithium glass scintillators. ${ }^{29}$

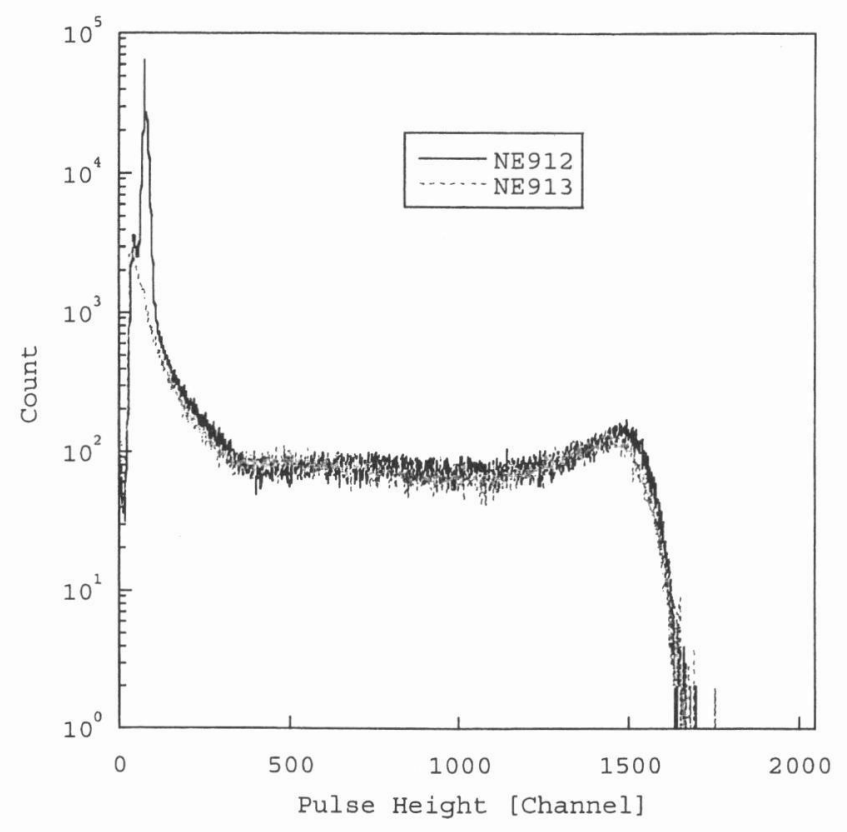

Figure 26. An example of the pulse height spectra of ${ }^{6} \mathrm{Li}$ and ${ }^{7} \mathrm{Li}$ glass scintillators. ${ }^{29}$

The neutron spectrum measurement was performed in the proton and neutron mixed field using the AVF cyclotron at NIRS. The protons accelerated up to $70 \mathrm{MeV}$ were irradiated onto a $2 \mathrm{~mm}$ thick beryllium target. The detector was placed at 45 degrees to the proton beam axis $350 \mathrm{~cm}$ behind the target. The protons passed through the target were stopped in an aluminum beam dump and the polyethylene blocks were put between the beam dump and the detector for neutron shielding from the dump. Figure 26 shows an example of the pulse height spectra of ${ }^{6} \mathrm{Li}$ and ${ }^{7} \mathrm{Li}$ glass scintillators mounted in 5 $\mathrm{cm}$ thick polyethylene. The ${ }^{6} \mathrm{Li}$ glass scintillator gives a large peak due to thermal neutrons through the ${ }^{6} \mathrm{Li}(\mathrm{n}, \alpha)$ reaction, while no peak can be found in the ${ }^{7} \mathrm{Li}$ glass scintillator. The thermal neutron events can easily be obtained from these two pulse height spectra. The counts measured by using five different moderators were unfolded with the SAND-2 code to get the neutron spectrum in this field. In this unfolding the $1 / E$ spectrum described before was also used as an initial guess spectrum. The unfolded spectrum is shown in Figure 27 together with the spectrum calculated by the MCNPX code for comparison. The spectrum measured with the detector gives rather good agreement with the calculation both in spectral shape and in absolute values.

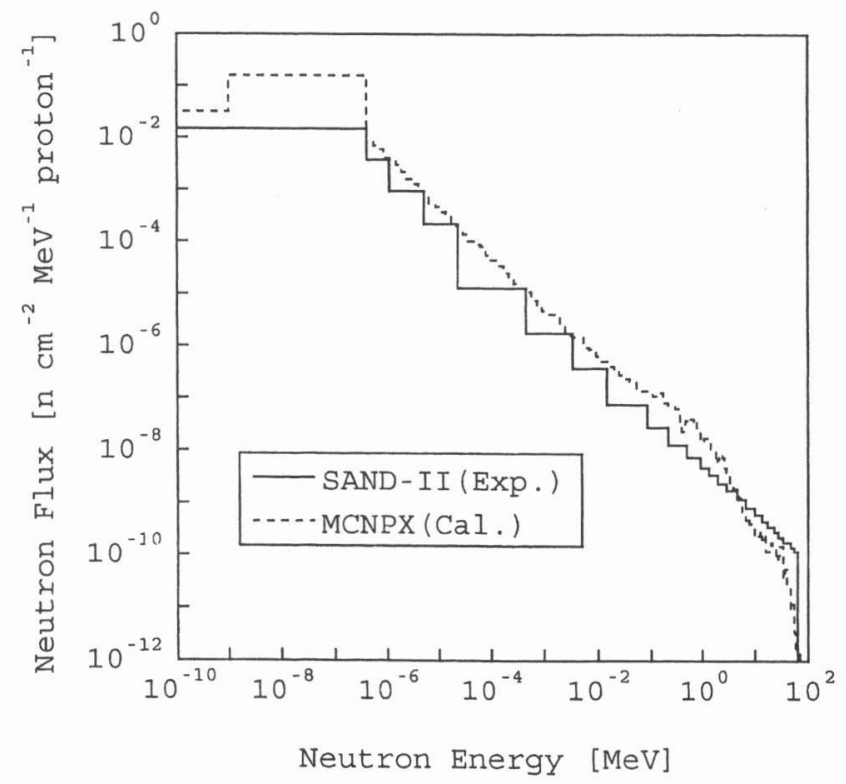

Figure 27. The unfolded spectrum with the spectrum calculated by the MCNPX code. ${ }^{29}$

\section{Summary}

For neutrons of energies beyond $100 \mathrm{MeV}$, the self-TOF detector using the NE102A plastic scintillators, large-scale NE213 organic liquid scintillator, and spallation detectors of C and $\mathrm{Bi}$ have been recently developed by our group, especially for use in neutron target and shielding experiments.

Various neutron spectrometers have also been developed for use in the charged particle and neutron mixed field to be realized in space environment. The following spectrometers are briefly described: 1) Phoswich detector which combines the NE115 plastic scintillator and the NE213 scintillator, 2) Anticoincidence detector system using $\Delta E$ and $E$ counters, and 3) Bonner sphere (multi-moderator detector) mounted a ${ }^{3} \mathrm{He}$ counter or a pair of ${ }^{6} \mathrm{Li}$ and ${ }^{7} \mathrm{Li}$ glass scintillators.

Our group has also developed a new-type Bonner sphere using a combination of polyethylene and boron moderators for measuring the neutron energy spectrum in the epithermal energy region of $1 \mathrm{eV}$ to $100 \mathrm{keV} .^{31}$ This detector will be used for neutron measurement in the accelerator-based boron neutron capture therapy.

These newly-developed detectors are now used in various applications. Further development of other advanced neutron spectrometers is now strongly required for increasing use of neutrons in various fields.

\section{REFERENCES}

(1) J. E. Keith, G.D. Badhwar, and D. J. Lindstrom, Nucl. Tracks. Radiat. Meas. 20, 41 (1992).

(2) V. Dudkin, Y. V. Potapov, A. B. Akopova, L. V. Melkumyan, E. V. Benton, and A. L. Frank, Nucl. Tracks. Radiat. Meas. 17, 87 (1990).

(3) A. P. Labakov, V. I. Lyagushin, M. I. Panasyuk, V. M. Petrov, and P. I. Shavrin, Nucl. Tracks. Radiat. Meas. 20, 55 (1992),

(4) N. Nakao, T. Nakamura, M. Baba, Y. Uwamino, N. Nakanishi, H. Nakashima, and Sh. Tanaka, Nucl. Instr. and Meth. A362, 454 (1995).

(5) N. Nakao, T. Kurosawa, T. Nakamura, and Y. Uwamino, Nucl. Instr. and Meth. A463, 275 (2001).

(6) M. Sasaki, N. Nakao, T. Nakamura, T. Shibata, and A. Fukumura, Nucl. Instr. and Meth. A480, 440 (2002).

(7) R. A. Cecil, B. D. Anderson, and R. Madey, Nucl. Instr. and Meth. 161, 439 (1979). 
(8) T. Moriya, Master Thesis of Kyoto University, March 2003, (in Japanese).

(9) E. Kim, T. Nakamura, A.Konno, Y. Uwamino, N. Nakanishi, M. Imamura, N. Nakao, T. Shibata, and Su. Tanaka, Nucl. Sci. Eng. 129, 209 (1998).

(10) Y. Uno, Y. Uwamino, T. S. Soewarsono, and T. Nakamura, Nucl. Sci. Eng. 122, 247 (1996).

(11) M. Baba, Y. Nauchi, T. Iwasaki, T. Kiyosumi, M. Yoshioka, S. Matsuyama, N. Hirakawa, T. Nakamura, Su. Tanaka, S. Meigo, H. Nakashima, Sh. Tanaka, and N. Nakao, Nucl. Instr. and Meth. A428, 454 (1999).

(12) N. Nakao, Y. Uwamino, T. Nakamura, T. Shibata, N. Nakanishi, M. Takada, E. Kim, and T. Kurosawa, Nucl. Instr. and Meth. A420, 218 (1999).

(13) Evaluated Nuclear Data File ENDF/B-VI (1990) National Neutron Cross Section Center, Brookhaven National Laboratory; P. F. Rose, ENDF-201, ENDF/B-VI Summary Documentation, BNL-NCS-17541, 4th Edition (1999).

(14)T. Fukahori, Proc. the 1991 Symposium on Nuclear Data, Tokai, Japan, JAERI-M 91-032, p106 (1991).

(15) M. Sasaki, M. Nakao, T. Shibata, N. Nakao, and T. Nakamura, Nucl. Instr. and Meth. A446, 545 (2000).

(16) M. Sasaki, N. Nakao, T. Nunomiya, T. Nakamura, T. Shibata, and A. Fukumura, J. Nucl. Sci. Technol. 38, 8 (2001).

(17) T. Kurosawa, T. Nakamura, N. Nakao, T. Shibata, Y. Uwamino, and A. Fukumura, Nucl. Instr. and Meth. A430, 400 (1999).

(18) R. E. Prael and H. Lichtenstein, User Guide to LCS: The LAHET Code System, MS B226, Los Alamos National Laboratory (1989).

(19) K. Shin, Y. Uwamino, and T.. Hyodo, Nucl. Technol. 53,
78 (1981).

(20) R. H. Johnson, FORIST: Neutron Spectrum Unfolding Code-Iterative Smoothing Technique, PSR-92, ORNL/ RSIC, Oak Ridge National Laboratory (1976).

(21) M. Takada, S. Taniguchi, T. Nakamura, and K. Fujitaka, IEEE Trans. on Nucl. Sci. 45, 888 (1998).

(22) M. Takada, S. Taniguchi, T. Nakamura, N. Nakao, Y. Uwamino, T. Shibata, and K. Fujitaka, Nucl. Instr. and Meth. A465, 498 (2001).

(23) T. Kurosawa, T. Nakamura, N. Nakao, T. Shibata, Y. Uwamino, and A. Fukumura, Nucl. Instr. and Meth. A430, 400 (1999).

(24) H. Matsumoto, T. Goka, K. Koga, S. Iwai, T. Uehara, O. Sato, and S. Takagi, Radiat. Meas. 33, 321 (2001).

(25) Y. Uwamino, T. Nakamura, and A. Hara, Nucl. Instr. and Meth. A239, 299 (1985).

(26) F. Judith, MCNP-A General Monte Carlo N-Particle Transport Code Version 4B, LA-12625-M, Los Alamos National Laboratory (1997).

(27) W. N. McElroy, S. Berg, T. Crockett, and R. G. Hawkins, A Computer-Automated Iterative Method for Neutron Spectra Determination by Foil Activation, AFWL-TR-6741, Air Force Weapons Laboratory (1967).

(28) S. Yamaguchi, A. Uritani, H. Sakai, C. Mori, T. Iguchi, H. Toyokawa, N. Takeda, and K. Kudo, Nucl. Instr. and Meth. A422, 600 (1999).

(29) S. Taniguchi, M. Takada, and T. Nakamura, Nucl. Instr. and Meth. A460, 368 (2001).

(30) L. S. Waters, ed. MCNPX ${ }^{\text {TM }}$ USER'S MANUAL, TPOE83-G-UG-X-00001 (1991).

(31) S. Yonai, T. Itaga, T. Nakamura, and M. Baba, J. Nucl. Sci. Technol. (in press). 